\section{A naturalist's evo-devo}

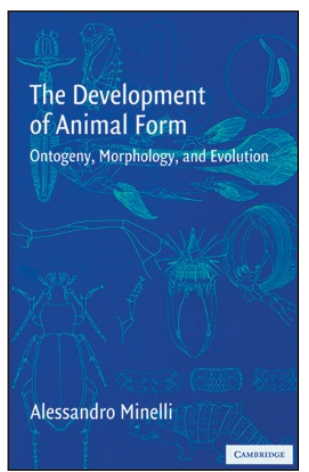

\section{The Development of Animal Form: Ontogeny, Morphology and Evolution}

by Alessandro Minelli

Cambridge University Press, $\$ 75.00342$

pp. hardcover ISBN 0-521-80851-0

\section{Reviewed by Michael Richardson}

Let's be honest: the much-hyped discipline of evolutionary developmental biology, or evo-devo, hasn't quite lived up to expectations-at least, not if we were expecting a revolution in biology. And when I visited the US recently, American colleagues told me that they have to purge the word 'Evolution' from their evo-devo grant applications. Their fear is that the 'E' word might alienate developmental biologists on the grant committee.

This is not to say that evo-devo is a flop. In several areas (Hox genes and the evolution of body plans, or the evolution of Amphioxus) evo and devo have been integrated to yield key new insights. But for the most part, evolutionary biology has been modestly enriched by evo-devo, whereas developmental biology has not. As an example, positional information theory, the leading idea in developmental biology in the last 35 years, has had minimal impact on evolutionary theory, except in the field of limb evolution. And by the same token, positional information remains a pre-darwinian concept because it did not borrow the comparative method from evolutionary biology.

How did we reach this state of affairs, and what can be done about it? As Alessandro Minelli argues in his important new book, a key problem is that the flow of ideas and data across the evo-devo bridge has been largely one way. For its part, evolutionary biology has willingly imported data and concepts from developmental biology and used them for phylogeny reconstruction and for attempting to enlighten such dark problems as homology. By contrast, says Minelli, potential exports of evolutionary biology, such as the comparative method, have largely failed to break into the developmental biology market.

Minelli's book, which is an enjoyable and stimulating read, aims to address these problems and to promote a freer trade in ideas across the evo-devo bridge. Minelli, a Professor of Zoology at Padua University in Italy, is well qualified to achieve the aims he sets for this book. He is an expert on arthropod biology, a leading zoological systematist and a rational and intelligent thinker. He is also a true naturalist, who brings to evo-devo a broadly based view of the animal kingdom.

The book is arranged around broad themes, such as 'segments' and 'axes and symmetries'. Minelli begins by trying to understand the nature of development itself. He believes that it is not simply a process for creating an adult but rather part of a cyclical continuum of life histories (would 'chicken and egg' be a better metaphor?). Anyhow, in this cyclical process, genes have not created form, but development has selected genes. Minelli's reasoning is that genetic networks that stabilize a particular morphology are strongly selected for, and genes are therefore selected by development. He even goes as far as to say ( $\mathrm{p}$. 24) that "the role of genes in morphogenesis is likely always to be an indirect one". This is probably going too far for most of us, however.

Minelli reviews arguments about the varied patterns of cell movement during vertebrate gastrulation. These patterns could have arisen, originally, from physical forces acting on the cells; later in evolutionary history, molecular genetic mechanisms could have been recruited to stabilize these cell movements. These ideas are fascinating and could help us to understand developmental homoplasy. My only concern here is that we may unwittingly be replacing universal genetic mechanisms with another set of universals: 'generic' cell properties. The problem with 'universals' is that they make for bad phylogenetics.

Minelli moves on to consider developmental stages and how they can be homologized across species. Evo-devo has generally ignored the problem of stages. Yet, until we are able to compare stages across species, comparative embryology cannot be a real science, and evodevo cannot become quantitative. If people studying Xenopus are using Nieuwkoop and Faber stages, and those studying the chick are using Hamburger and Hamilton, then we are trying to compare Betamax and VHS. Minelli does miss a point, however: one reason heterochrony is interesting to developmental biologists is that shifts in timing can produce key changes in morphology if the patterning mechanism involved has a temporal component.

The book touches on other important but neglected topics, such as complexity, size and miniaturization, and the origin of the tail. Minelli also devotes a fascinating section to the commonality of patterning mechanisms between limbs and genitalia in different phyla. The chapter on segments is a tour de force in terms of its breadth of coverage and reveals Minelli's gift for integrating data and ideas from disparate sources (including his own research).

I hope people will read this book, because it contains much of value and interest. Some readers of Nature Genetics may take issue with Minelli's rejection of what he calls the 'gene-centered' view of development that has come to dominate in recent years; some may even see Minelli as a morphologist swimming against the irresistible tide of genetics and molecular developmental biology. Minelli denies this, however, and my reading of the book is that it is a genuine effort to reconnect evo-devo with a broader natural history. In this, it succeeds beautifully. 